\title{
Monitoring The After-Effects Of Ischemic Stroke Through EEG Microstates And Machine Learning
}

\section{Fang Wang}

XiHua University

Yu-Chu Tian ( $\nabla$ y.tian@qut.edu.au )

Queensland University of Technology

Xueying Zhang

Taiyuan University of Technology

Fengyun $\mathrm{Hu}$

Shanxi Provincial People's Hospital affiliated with Shanxi Medical University

\section{Research Article}

Keywords:

Posted Date: February 16th, 2022

DOI: https://doi.org/10.21203/rs.3.rs-1297608/v1

License: (c) (1) This work is licensed under a Creative Commons Attribution 4.0 International License. Read Full License 


\title{
Monitoring the after-effects of Ischemic Stroke through EEG Microstates and Machine Learning
}

\author{
Fang Wang ${ }^{1,2}$, Yu-Chu Tian ${ }^{3, *}$, Xueying Zhang ${ }^{4, *}$, and Fengyun $\mathrm{Hu}^{5, *}$ \\ ${ }^{1}$ School of Comprehensive Health Management, XiHua University, Chengdu, 611730, China \\ ${ }^{2}$ West China Biomedical Big Data Center of West China Hospital, Sichuan University, Chengdu, 610041, China \\ ${ }^{3}$ School of Computer Science, Queensland University of Technology, QLD 4059Australia \\ ${ }^{4}$ College of Information and Computer, Taiyuan University of Technology, Taiyuan, 030606, China \\ ${ }^{5}$ Department of Neurology, Shanxi Provincial People's Hospital affiliated with Shanxi Medical University, Taiyuan, \\ 030012, China \\ *Yu-Chu Tian: y.tian@qut.edu.au; Xueying Zhang: tyzhangxy@163.com; Fengyun Hu: fengyun71@163.com
}

\begin{abstract}
Background and Purpose:Stroke may cause extensive after-effects such as motor function impairments and disorder of consciousness (DoC). Detecting these after-effects of stroke and monitoring their changes are challenging jobs currently undertaken via traditional clinical examinations. These behavioural examinations often take a great deal of manpower and time, thus consuming significant resources. Computer-aided examinations of the electroencephalogram (EEG) microstates derived from bedside EEG monitoring may provide an alternative way to assist medical practitioners in a quick assessment of the after-effects of stroke. Methods:In this study, we designed a framework to extract microstates maps and calculate their statistical parameters to input to classifiers to identify DoC in ischemic stroke patients automatically. As the dataset is imbalanced with the minority of patients being DoC, an ensemble of support vector machines (EOSVM) is designed to solve the problem that classifiers always tend to be the majority classes in the classification on an imbalanced dataset. Results: The experimental results show EOSVM get better performance (with accuracy and F1-Score both higher than $89 \%$ ), improving sensitivity the most, from lower than $60 \%$ (SVM and AdaBoost) to higher than $80 \%$. This highlighted the usefulness of the EOSVM-aided DoC detection based on microstates parameters. Conclusion: Therefore, the classifier EOSVM classification based on features of EEG microstates is helpful to medical practitioners in DoC detection with saved resources that would otherwise be consumed in traditional clinic checks.
\end{abstract}

\section{Introduction}

Stroke is one of the most prevalent neurological conditions worldwide ${ }^{1}$. It is also a leading cause of severe disability ${ }^{2}$, such as extensive motor function impairment, cognitive disorders, and disorder of consciousness (DoC). Detecting these after-effects of stroke and monitoring the changes in the clinical condition of patients are challenging jobs. They are currently undertaken via traditional behavioral and clinical examinations, which carry a high test-retest and inter-examiner variability ${ }^{3}$. Any changes in the clinical states of a patient cannot always be identified in a timely manner. Their detection largely relies on how long the interval is to the next clinical examination. Furthermore, significant manpower, time and other resources are consumed in these clinical examinations for both inpatients and outpatients. For stroke patients, pathological changes of certain areas of the brain can cause motor function impairment. Identification of motor disturbance in poststroke patients timely is necessary ${ }^{4,5}$.

Resting-state electroencephalography (EEG) monitoring provides an alternative way to potentially assist medical practitioners in a quick assessment of the after-effects of stroke. EEG studies have shown that there are brief periods in which global electrical brain activities on the scalp remain semi-stable ${ }^{6}$. Microstates analysis segments EEG data into a limited number of clusters with a duration varying between $40-150 \mathrm{~ms}$ based on global points ${ }^{7}$. These transient periods of stability have different topographical representations, namely microstates. EEG Microstate analysis has been increasingly investigated for the spatial and temporal properties of whole-brain neuronal networks ${ }^{7}$.

As a tool for the study of brain activities, EEG Microstates have been applied in the investigation of neuropsychiatric diseases. A study in NeuroImage examined the degree to which spatial and temporal properties of microstates might be altered by manipulating cognitive task (a serial subtraction task versus wakeful rest). It provided visual information (eyes open versus eyes closed conditions) to medical practitioners ${ }^{6}$. Another study demonstrated that altered states of consciousness, e.g., sleep, hypnosis, and meditation, were correlated with changes in microstate properties ${ }^{8}$. Other studies explored EEG microstate changes in neurological diseases, such as schizophrenia ${ }^{9-11}$, head injury ${ }^{12}$, dementia ${ }^{10}$, and narcolepsy ${ }^{13}$.

Many investigations have been undertaken on how the properties of EEG microstates vary across different cognitive tasks, 
genders, medications, and diseases. In spite of these efforts, the challenge of microstate analysis is to design experiments that are capable of establishing direct causal relations between the EEG microstates and certain hypotheses ${ }^{7}$. Particularly, only one report was found so far on the examination of EEG microstates in stroke patients ${ }^{14}$. It compared the parameters of EEG microstates derived from 47 stroke patients and 20 healthy controls in three groups (the left hemisphere lesion stroke group, the right hemisphere lesion stroke group, and the healthy controls group).

Our work in this paper makes two main contributions:

1. The first contribution is our establishment of correlations between EEG microstates with the clinical states of stroke patients through experimental studies of 152 patients. We designed an experimental procedure to extract microstate maps from a single dataset aggregated from multiple EEG datasets of all patients. Then, we investigated the correlations between EEG microstates with the level of DOC (awake, somnolence, stupor, light coma, middle coma, and deep coma) through the Spearman correlation coefficient. Moreover, an one-way ANOVA analysis was carried out to investigate the differences of EEG microstate parameters between different motor disturbance groups.

2. The second contribution is our design of a classifier for the detection of the $\mathrm{DoC}$ of stroke patients. We employed an ensemble of support vector machines (EOSVM) as the framework of the classifier. EEG microstates together with the statistical microstate parameters were input to the classifier. We compared the performance of the EOSVM under different numbers of microstate maps and different settings of the EOSVM majority voting.

We used two widely accepted classifiers as base models in our experiments: a single SVM classifier and an AdaBoost classifier ${ }^{15,16}$.

The rest of this paper is organized as follows: Section presents materials, procedures of EEG microstate analysis, and the framework of our EOSVM classifier. Our main results are summarized in Section on correlation analysis between microstates and level of DoC in stroke patients, and Section on comparing classification results from EOSVM with Support Vector Machine (SVM) and Adaptive Boosting (AdaBoost). Further discussions on the experimental results are given in Section . Finally, Section concludes the paper.

\section{Materials and Methods}

\section{Participants}

There were 152 stroke patients (mean age $=64.76$ years, standard deviation $(\mathrm{SD})=15.63$ years) in this study. These subjects were patients admitted to the neurology department at Shanxi Provincial People's Hospital after acute stroke from 2017 to 2018. Table 1 summarized the demographics and clinical characteristics of the participants. Among the 152 stroke patients, Inclusion criteria were as follows: (1) The patients were diagnosed with ischemic stroke, (2) EEG data were recorded and available for analysis, and (3) the corresponding assessment of consciousness by medical practitioners were recorded. Exclusion criteria were: (1) patients younger than 18 years old, and (2) pregnant patients. All data were collected as part of a prospective observational cohort study approved by the local institutional review board of the Shanxi Provincial People's Hospital and informed consent was obtained from all subjects or their legal guardian. All methods in this study were carried out in accordance with relevant guidelines and regulations.

Table 1. Demographics and clinical characteristics of 152 patients.

\begin{tabular}{lccc}
\hline $\begin{array}{l}\text { State of } \\
\text { Consciousness }\end{array}$ & $\begin{array}{c}\text { Number of } \\
\text { Patients }\end{array}$ & $\begin{array}{c}\text { Female } \\
\text { Patients }\end{array}$ & $\begin{array}{c}\text { Age } \\
\text { (Mean, SD) }\end{array}$ \\
\hline Awake & 95 & 24 & $63.05,15.31$ \\
Somnolence & 29 & 9 & $69.17,18.17$ \\
Stupor & 14 & 5 & $65.29,11.22$ \\
Light coma & 7 & 2 & $70.71,16.64$ \\
Middle coma & 6 & 2 & $60.33,13.94$ \\
Deep coma & 1 & 0 & $76.00,0.00$ \\
\hline OVERALL & 152 & 42 & $64.76,15.63$ \\
\hline
\end{tabular}

The state of consciousness of each patient was determined by using a hierarchical battery of observation assessments. The assessment method of consciousness has been summarized in our previous study ${ }^{17}$. For all patients, neurological examinations with the assessments of consciousness were performed before EEG signals were recorded. All clinical assessments were performed by medical practitioners who were blinded to the EEG measures of the patients. 


\section{EEG Data Acquisition and Pre-processing}

EEG signals were recorded through a bedside digital video EEG monitoring system (Solar 2000 N, Solar Electronic Technologies Co., Ltd, Beijing, China) at a sampling rate of $100 \mathrm{~Hz}$. The specific electrodes sites were positioned at FP1, FP2, C3, C4, O1, $\mathrm{O} 2, \mathrm{~T} 7, \mathrm{~T} 8, \mathrm{~A} 1$, and A2 according to the international 10-20 system. EEG data were recorded continuously for at least 2 hours and impedance was established below $10 \mathrm{k} \Omega$ for all electrode sites. The maximum allowed interval between the end of the clinical examination and the start of the EEG recording time was 30 minutes in this study.

Our EEG pre-processing was carried out offline in MATLAB (Mathworks, Natick, MA) with the EEGLAB toolbox (version 14.1.1b). The continuous EEG data were first re-referenced to an average reference, before which bad channel has been rejected. Then, high-pass filtering $(0.5 \mathrm{~Hz})$ and low-pass filtering $(40 \mathrm{~Hz})$ were successively applied to the EEG signals by using a basic finite impulse response (FIR) filter. For the resulting EEG signals, we detected artefacts based on eye movements, muscle activity, and amplitude threshold violations $(150 \mathrm{~V})$. The trials with any of these artefacts were removed.

\section{Microstate analysis}

Our microstate analysis followed the procedure in Figure 1. Briefly speaking, the clean and processed EEG data recorded in the first five minutes were included in the microstate analysis for each subject. The EEG datasets from all the 152 stroke subjects were aggregated into one dataset. This dataset was then used to derive microstate prototypes. After that, these microstate prototypes were back-fit to EEG data from each subject. After the EEG microstates were defined for each of the patients, statistical temporal parameters were calculated from the derived datasets. The microstates in this study were extracted through a plugin for EEGLAB, Microstate EEGLAB toolbox ${ }^{18}$.

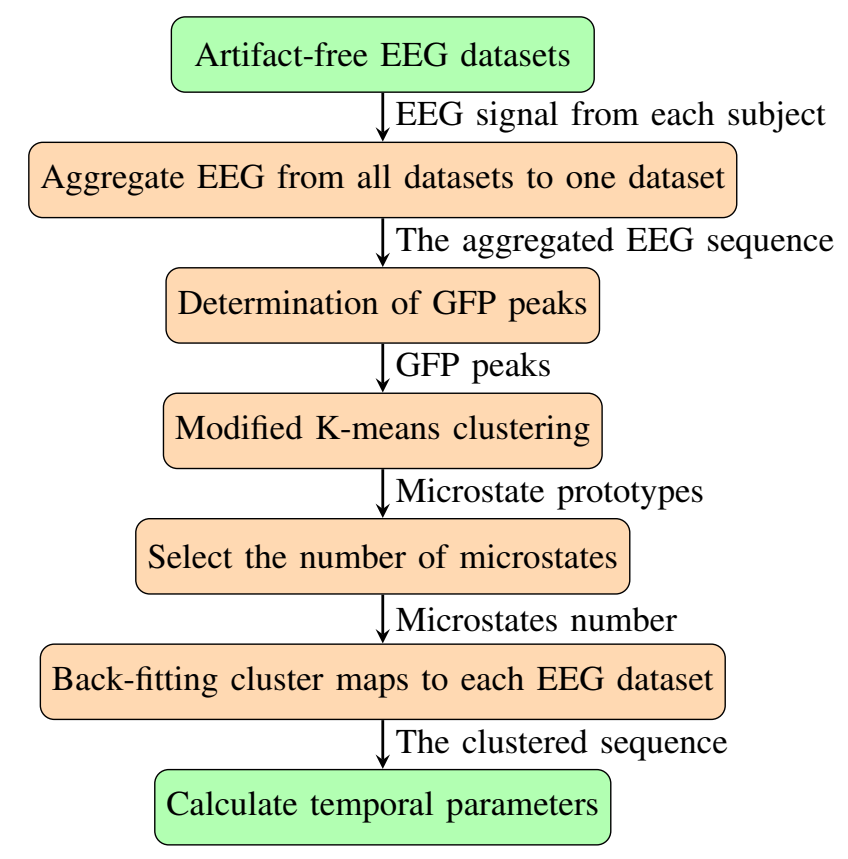

Figure 1. The procedure of the microstate analysis in this study.

\section{Microstate segmentation}

Here, we explain how to derive microstate prototypes from the aggregated dataset. Firstly, the global field power (GFP), which is the spatial standard deviation of EEG signals across all channels, was calculated. Then, a clustering method was used to group the GFP sequences into a small set of classes based on topographic similarity. We used a modified k-means method in this study. Each of the resulting clusters could describe a topographical prototype, namely a microstate prototype.

The Modified k-means method adds several features to clustering ${ }^{19}$. Here the modified k-means models introduce two differences compared to conventional k-means. The first is that the topographical maps of prototypical microstates with opposite polarity are assigned to the same cluster. The second difference is that modified k-means models the activations of the microstates i.e. models the strength of the microstates for each time point ${ }^{20}$. 


\section{Microstate parameters}

Microstate parameters, duration, occurrence, coverage, and variance were calculated in the following for the sorted microstates $^{6,18}$ :

1. Duration: the average duration that a given microstate remains stable.

2. Occurrence: the number of times a microstate occurred during one second.

3. Coverage: the total percentage of time covered by a given microstate.

4. Variance: global explained variance (GEV) is a measure of how similar each EEG sample is to the microstate prototype that the sample has been assigned to ${ }^{20}$. The higher the GEV is, the higher the similarity is.

\section{Classification Framework}

In general, datasets from a hospital, such as EEG signals, are imbalanced. For EEG signals from stroke patients, the datasets consist of much more wakeful samples than DoC ones. A common problem in training a classifier from imbalanced datasets is that the trained classifier is more likely to predict a sample as the majority class. This will lead to critical false negatives when DoC is considered as positive.

To address this problem, we designed a classifier of an ensemble of support vector machines (EOSVM) formed from multiple SVM classifiers, as shown in Figure 2. In the training phase, the overall datasets for training are aggregated and then split into $N$ subsets. These $N$ subsets are fed into $N$ SVMs, respectively. In the test phase, all SVMs take the same input for prediction. Each SVM makes its own decision, which classifies the corresponding sample into wakefulness or a DoC state. After that, multiple predictions from the $N$ SVMs are fused through a voting rule to make the final prediction. In this study, we use a simple majority voting rule.

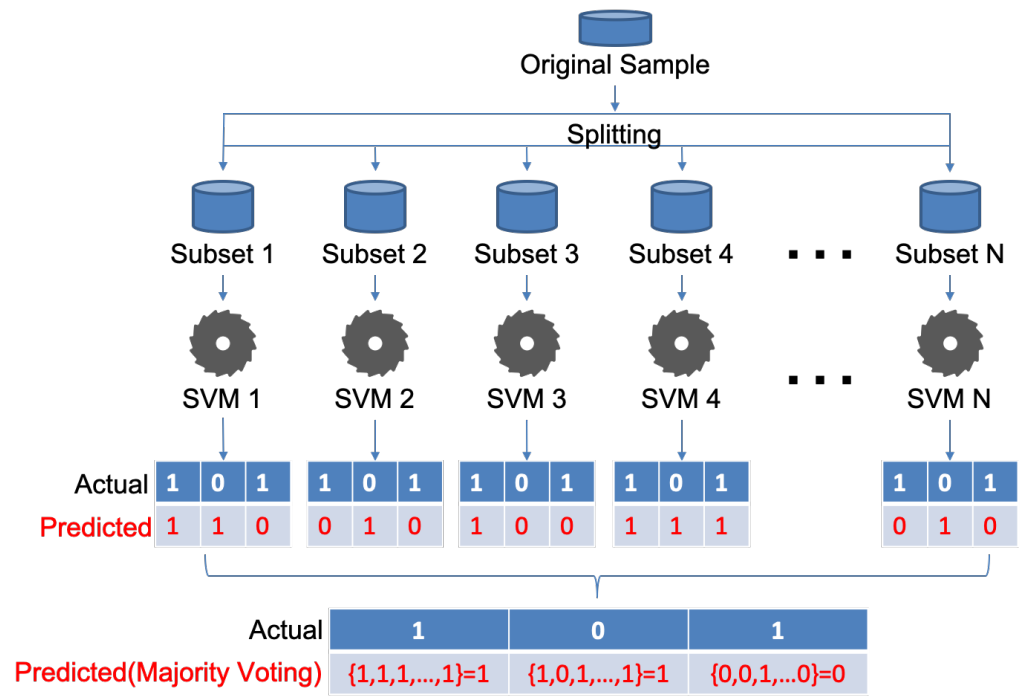

Figure 2. The framework of our EOSVM classifier. In the training phase, each of the SVMs in the EOSVM takes a different subset of data as its input as shown in the upper part of the figure. In the test phase, all SVMs take the same input for prediction, for which an example of 3 samples is shown in the lower part of the figure.

The number of SVMs in the EOSVM classifier should be adjusted according to the distribution of a dataset. Normally, the more heavily imbalanced the dataset is, the more SVMs should be used in the EOSVM classifier. However, the largest number of SVMs in EOSVM should be capped by the number of combinations of $r$ objects chosen from $n$ objects as follows:

$$
C(n, r)=\frac{n !}{r !(n-r) !}
$$

where $r$ represents the number of the minority classes in the training dataset, and $n$ represents the number of the majority classes. In this study, the minority classes are the DoC classes, while the majority classes are the awakeness classes.

The classification of the EEG signals are performed in three steps: 1) data aggregation and splitting, 2) classifier training (in the training phase) and prediction, and 3) voting for final prediction. These steps are already shown in Figure 2. They are described below in more detail. 


\section{Data aggregation and splitting}

We randomly split the entire dataset into a training set ( $80 \%$ of the data) and a test set ( $20 \%$ of the data). In order to train the $N$ SVMs in the EOSVM classifier, we further build $N$ balanced training subsets from the training set. For each of the subset, we first include all the DoC samples from the training set since they only account for a small proportion of the training set, and then randomly select the same number of wakefulness samples from the training set to add to the training subset. Each training subset and the test set make up a subset as an input to an SVM.

\section{Classifier training and prediction}

To train each of the $N$ SVM classifiers, we employ the Gaussian kernel function. In the training, each SVM automatically tunes the capacity of the classification function by maximizing the margin between training examples and class boundary, optionally after removing some atypical or meaningless examples from the training data. The hyper-parameters are obtained by maximizing the marginal.

For prediction, the $N$ SVMs make their respective decisions to diagnose in or out of either wakefulness or DoC of the patients.

\section{Voting for final prediction}

The $N$ predictions from the $N$ SVMs may be the same or different. They are fused through a voting rule. In standard majority voting, the two-class with the more votes from the $N$ SVMs is determined as the final classification result. However, if the votes for class 1 is only more than the votes for class 2 a little, e.g., 51 versus 49 , we only have low confidence for the classification result of the voting result. If the votes for one class is much higher than the other, e.g., 88 versus 11, the final classification is more clinically reliable. Therefore, only explore the voting threshold of 50\% is not enough. We tested over $50 \%$ majority voting up to $100 \%$ majority voting in the experiments. Form our test experiments, some insights were developed on the number of EEG microstates for the best prediction performance, and the threshold of the majority voting for the best prediction results.

\section{Evaluation of Classification Performance}

To evaluate the performance of classification and prediction, we use two groups of metrics. The first group is introduced from machine learning and classification perspectives, while the second group is more on the medical assessment.

The first group of metrics includes Accuracy, F1-Score, Sensitivity, and Specificity, which are common metrics for classification assessment in machine learning. We consider subjects with DoC as positive cases in this study. Let TP, TN, FP, FN denote true positives, true negatives, false positives, and false negatives, respectively.

These four metrics are mathematically describes as:

$$
\begin{aligned}
& \text { Accuracy }=\frac{T P+T N}{T P+T N+F P+F N} \times 100 \% \\
& \text { Sensitivity }=\frac{T P}{T P+F N} \times 100 \% \\
& \text { Specificity }=\frac{T N}{T N+F P} \times 100 \% \\
& F 1 \text {-Score }=\frac{2 T P}{2 T P+F P+F N} \times 100 \%
\end{aligned}
$$

The physical meanings of these four metrics are described below. The performance of accuracy characterizes the percentage of true classifications overall classified subjects. Sensitivity refers to the ability to identify true positives among all subjects who have been predicted as positive, i.e., the patients who diagnosed with DoC do have DoC. Specificity quantifies the ability to detect true negatives among all subjects who have been predicted as negative, i.e., the patients diagnosed with no DoC do have no DoC. It helps rule out those patients without DoC. Moreover, F1-Score is the harmonic mean of precision and sensitivity.

While the above four metrics in the first group are useful in evaluating the overall performance of the EOSVM performance, clinical assessments of patients require more certain results. For example, given a classification accuracy of $90 \%$, what can we say if a patient is predicted by the classifier to have DoC? Likely, the patient has the DoC. But this is not enough in a medical assessment. We need to know if the patient really has DoC or not.

Therefore, three additional metrics are designed to characterize the capability of the EOSVM classifier to predict assessment results more relevant to clinic diagnosis. They form the second group of our metrics. They are specified in this study as True Prediction, False Prediction, and Not Sure (for those cases the EOSVM classifier is unable to give a certain prediction useful for clinic examinations).

Before calculating True Prediction, False Prediction, and Not Sure, we need to define Prediction Rate as a metric to evaluate the percentage of subjects with a prediction among all subjects. It is formulated as:

$$
\text { PredictionRate }=\frac{T P+F P+T N+F N}{N_{a w a}+N_{D o C}} \times 100 \%
$$


where $N_{D o C}$ and $N_{a w a}$ are the total number of positive subjects (DoC) and negative subjects (no DoC), respectively.

Then the second group of metrics are mathematically describes as:

$$
\begin{aligned}
& \text { TruePrediction }=\text { Accuracy } \times \text { PredictionRate } \\
& \text { NotSure }=(1-\text { PredictionRate }) \times 100 \% \\
& \text { FalsePrediction }=(1-\text { TruePrediction }- \text { NotSure }) \times 100 \%
\end{aligned}
$$

It is worth mentioning that the sum of TP, TN, FP, and FN is not equal to $N_{a w a}+N_{D o C}$. This is because some subjects may have no final predictions for some settings of the majority voting in EOSVM.

\section{Statistical Analysis}

Firstly, Spearman correlations between the level of consciousness and microstate parameters were employed to explore the relationship between consciousness and microstates. Secondly, an independent T-test was used to determine significant differences between different motor disturbance groups for investigating the relationships between motor impairment and microstates. Finally, we analyzed the statistics of the classification results.

\section{Results on Statistical Analysis}

Here, we analyzed more than four primary microstates prototypes coming from the clustering. For the number of clusters of microstates, previous experimental and clinical studies focused on four primary cluster maps, which were labeled as classes $\mathrm{A}, \mathrm{B}, \mathrm{C}$, and $\mathrm{D}^{6,8,13,21-23}$. Ideally, the best number of clusters should be estimated for each dataset individually using robust optimization criteria, rather than a fixed value ${ }^{7,20}$. Considering the extracted microstates in our dataset, we investigated various scenarios with a varying number of microstate maps. For six microstates, e labeled them as A, B, C, D, E and F as shown in Figure 3. These six microstates were also explored by previous studies ${ }^{7,24}$. Additionally, we also explored the scenarios when EEG data were clustered to 2, 4, 6, 8, 10, and 12 microstate maps, as shown in Figure 4.

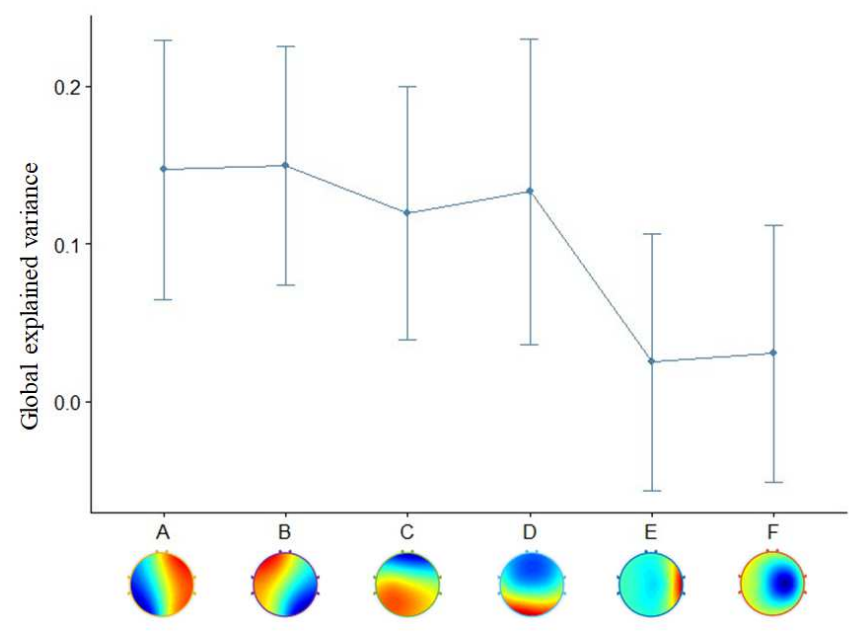

Figure 3. Microstate prototypes and their Global explained variance (GEV) for the scenario with 6 clusters. GEV is a measure of how similar each EEG sample is to the microstate prototype it has been assigned to ${ }^{20}$. In the correlation analysis and T-test in this study, we focused on these 6 cluster maps as in some previous studies.

\section{Microstates and the Level of Consciousness}

For exploring whether the microstates parameters (duration, occurrence and coverage) can be used as features to classify the different levels of consciousness, we analyzed the correlation between microstates parameters with the level of consciousness. In clinic examinations, there are six levels of consciousness (L6), i.e., wakefulness, somnolence, stupor, light coma, middle coma, and deep coma. They are numbered from 1 to 6 , respectively. The bigger the numerical value of the level is, the worse the state of consciousness is. Spearman correlation coefficients are calculated between microstate parameters and the level of consciousness. The results are shown in Figure 5.

The first columns of both correlation matrices in Figure 5 reveal the relationships between 18 EEG features and the level of consciousness. The 18 EEG features are the three microstate parameters, duration, occurrence and coverage from the six 


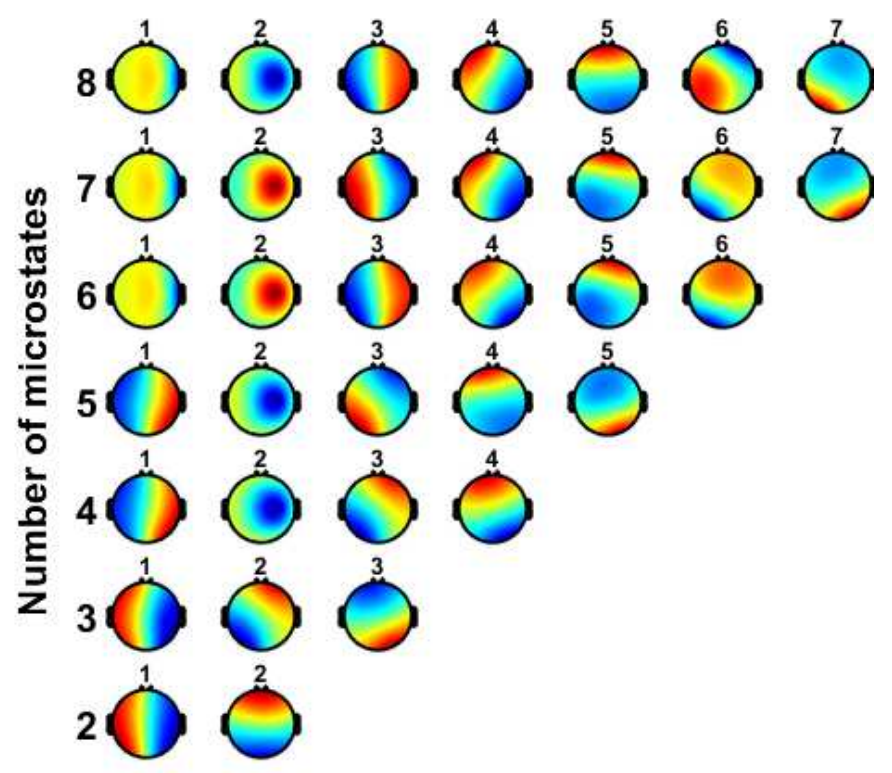

Figure 4. Microstate prototypes of spatial clustering analysis using a modified K-means clustering method. The clustering analysis of the maps was carried out at the GFP peaks of the EEG dataset aggregated from all EEG files of 152 subjects. The graph shows the cluster maps when EEG data were clustered to $k$ microstates for $k=2,3, \cdots, 8$.

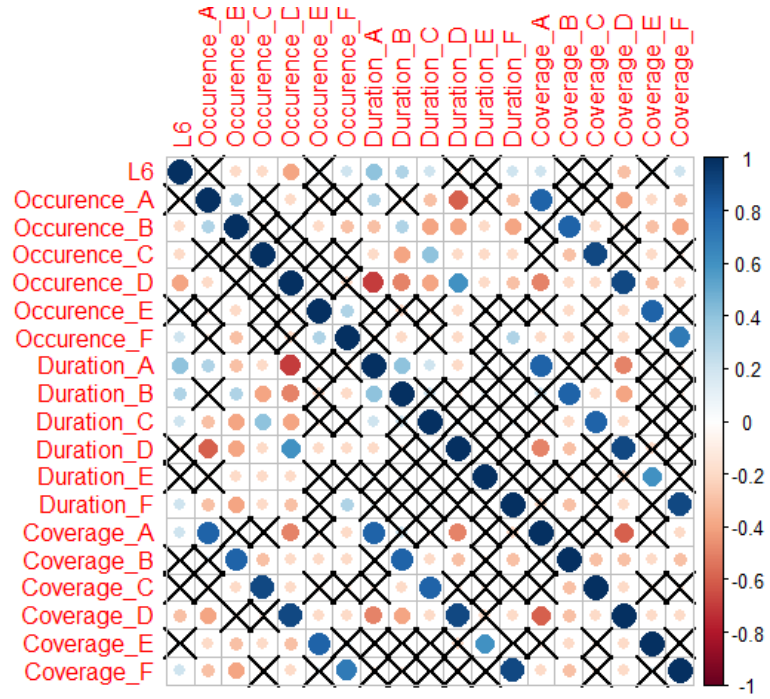

(a)

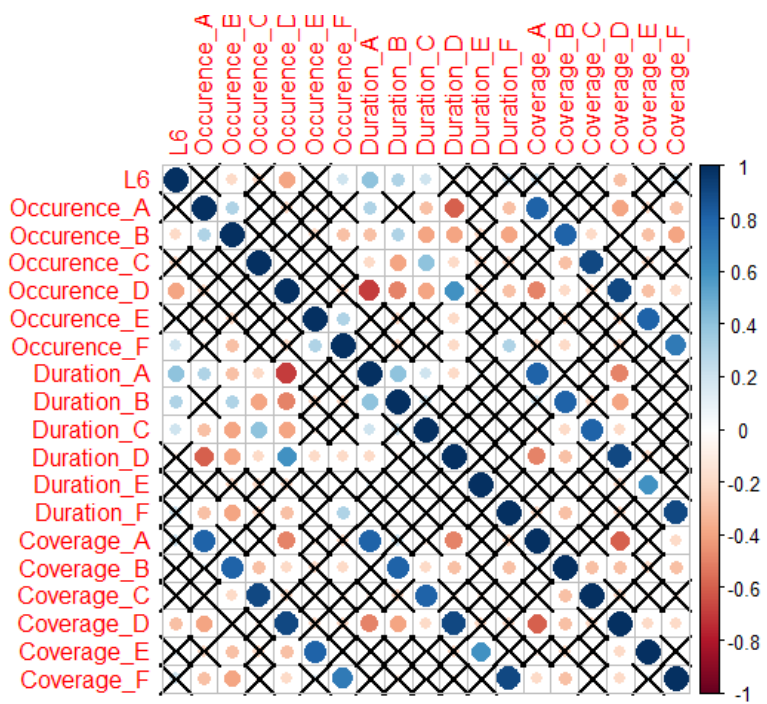

(b)

Figure 5. Correlation matrices visualized with colored significance levels of correlations between EEG microstates and the level of consciousness in stroke subjects. The color 'blue' represents positive correlations and color 'red' refers to negative correlations. In the subfigure (a), correlations with $p$-value $<0.05$ are considered to be significant and the insignificant ones are marked with ' $\times$ '. Subfigure (b) are correlations with $p$-value $<0.01$.

different microstates A, B, C, D, E and F. Let us focus on the four microstates A, B, C, and D first, which have been previously examined in the literature. It is seen from Figure 5 that the 'occurrences' of microstate $\mathrm{B}(P<0.01), \mathrm{C}(P<0.05)$, and $\mathrm{D}$ $(P<0.01)$ were significantly correlated with the level of consciousness in stroke patients. For parameter 'duration', microstates $\mathrm{A}, \mathrm{B}$, and $\mathrm{C}$ had significant correlations with the level of consciousness with a positive trend $(P<0.01)$. The 'coverage' of microstates A $(P<0.05)$ and $\mathrm{D}(P<0.01)$ were also significantly correlated with the level of consciousness in stroke subjects (with positive and negative correlations, respectively).

If we focused on microstates $\mathrm{E}$ and $\mathrm{F}$, it was observed from Figure 5 that microstate $\mathrm{E}$ did not show significant correlations 
with the level of consciousness in all the three statistical parameters 'occurrence', 'duration' and 'coverage'. However, microstate $\mathrm{F}$ was significantly correlated with the level of consciousness with a positive trend in all the three statistical parameters.

\section{Microstates and Motor Impairment}

We investigated whether there were any statistically significant differences of microstate parameters among four groups through the one-way analysis of variance (ANOVA). The four groups are the left motor disturbance (41 subjects), the right motor disturbance ( 45 subjects), the bilateral motor disturbance (6 subjects), and no motor disturbance (57 subjects). Among the total 152 subjects in this study, 3 subjects have no information about motor impairment. Thus, 149 subjects are included in the motor impairment analysis. Table 2 revealed the mean and standard deviation (SD) of the microstates parameters. It also showed the results of the one-way ANOVA and the following post hoc test determining which of the four groups differ from each other.

Table 2. Microstate characteristics, between-group ANOVA ( $\mathrm{df}=3)$, and multiple comparisons (LSD post hoc test). Significant differences $(p<0.05)$ are displayed in bold type.

\begin{tabular}{|c|c|c|c|c|c|c|c|c|c|c|}
\hline \multirow[t]{2}{*}{ Microstate } & \multicolumn{4}{|c|}{ Descriptives (Mean, SD) } & \multicolumn{2}{|c|}{ ANOVA } & \multicolumn{4}{|c|}{ Post-hoc comparisons - $p$ value } \\
\hline & No(57) & Left(41) & Right(45) & Bilateral(6) & $\mathrm{F}$ & $p$ & $\overline{\mathrm{L} v s \mathrm{R}}$ & $\mathrm{N}$ vs L & $\mathrm{N}$ vs R & $\mathrm{N}$ vs $\mathrm{B}$ \\
\hline \multicolumn{11}{|l|}{ Occurrence } \\
\hline $\mathrm{A}$ & $4.59,0.90$ & $4.37,1.22$ & $5.10,0.82$ & $4.91,0.93$ & 4.411 & 0.005 & 0.001 & 0.272 & 0.010 & 0.444 \\
\hline B & $4.76,0.96$ & $4.56,1.04$ & $4.97,0.78$ & $4.88,0.66$ & 1.457 & 0.229 & 0.040 & 0.273 & 0.268 & 0.776 \\
\hline $\mathrm{C}$ & $3.78,1.05$ & $3.65,0.84$ & $3.90,0.90$ & $4.04,0.41$ & 0.626 & 0.599 & 0.230 & 0.492 & 0.550 & 0.532 \\
\hline $\mathrm{D}$ & $4.08,1.24$ & $4.59,1.42$ & $4.33,1.19$ & $4.59,1.55$ & 1.352 & 0.260 & 0.351 & 0.055 & 0.330 & 0.361 \\
\hline $\mathrm{E}$ & $1.61,0.85$ & $1.69,0.81$ & $1.64,0.84$ & $1.33,0.57$ & 0.361 & 0.781 & 0.771 & 0.618 & 0.844 & 0.432 \\
\hline $\mathrm{F}$ & $2.14,0.96$ & $1.93,0.83$ & $1.96,0.74$ & $1.70,0.28$ & 0.896 & 0.445 & 0.869 & 0.226 & 0.287 & 0.227 \\
\hline \multicolumn{11}{|l|}{ Duration } \\
\hline A & $49.64,9.54$ & $49.17,12.67$ & $49.40,7.90$ & $50.51,11.67$ & 0.039 & 0.990 & 0.915 & 0.819 & 0.905 & 0.843 \\
\hline B & $50.94,9.95$ & $47.73,7.71$ & $47.64,5.96$ & $46.96,3.91$ & 1.948 & 0.124 & 0.958 & 0.056 & 0.044 & 0.256 \\
\hline $\mathrm{C}$ & $47.53,10.96$ & $44.98,7.48$ & $43.00,7.22$ & $44.69,7.16$ & 2.215 & 0.089 & 0.305 & 0.164 & 0.012 & 0.459 \\
\hline D & $46.88,9.53$ & $51.71,10.76$ & $45.41,9.17$ & $48.21,13.69$ & 3.141 & 0.027 & 0.004 & 0.019 & 0.460 & 0.757 \\
\hline $\mathrm{E}$ & $35.24,8.37$ & $35.15,6.80$ & $39.26,44.25$ & $31.16,4.81$ & 0.352 & 0.788 & 0.451 & 0.986 & 0.425 & 0.706 \\
\hline F & $36.89,8.14$ & $39.54,27.16$ & $33.20,4.03$ & $32.29,1.83$ & 1.395 & 0.247 & 0.057 & 0.398 & 0.229 & 0.485 \\
\hline \multicolumn{11}{|l|}{ Coverage } \\
\hline $\mathrm{A}$ & $0.23,0.07$ & $0.22,0.09$ & $0.26,0.07$ & $0.25,0.10$ & 1.830 & 0.144 & 0.034 & 0.574 & 0.085 & 0.443 \\
\hline B & $0.24,0.08$ & $0.22,0.07$ & $0.24,0.06$ & $0.23,0.04$ & 1.007 & 0.392 & 0.230 & 0.092 & 0.663 & 0.619 \\
\hline $\mathrm{C}$ & $0.18,0.07$ & $0.17,0.05$ & $0.17,0.06$ & $0.18,0.04$ & 0.730 & 0.536 & 0.714 & 0.169 & 0.309 & 0.921 \\
\hline D & $0.20,0.08$ & $0.25,0.12$ & $0.20,0.10$ & $0.24,0.15$ & 2.377 & 0.072 & 0.049 & 0.013 & 0.668 & 0.353 \\
\hline $\mathrm{E}$ & $0.06,0.05$ & $0.06,0.04$ & $0.06,0.05$ & $0.04,0.03$ & 0.279 & 0.841 & 0.983 & 0.899 & 0.915 & 0.400 \\
\hline $\mathrm{F}$ & $0.08,0.06$ & $0.08,0.09$ & $0.07,0.03$ & $0.06,0.01$ & 0.937 & 0.425 & 0.264 & 0.906 & 0.183 & 0.289 \\
\hline
\end{tabular}

For microstate parameter occurrence rate of microstate A, result of one-way ANOVA found that there was statistically significant difference between groups, $\mathrm{F}(3,145)=4.411, p=0.005<0.05$. A LSD post hoc test revealed that occurrence of microstate A was statistically significantly lower in the left motor disturbance group $(4.37 \pm 1.22, p=0.001)$ and no motor disturbance group $(4.59 \pm 0.90, p=0.010)$ compared to the right motor disturbance group $(5.10 \pm 0.82)$ which are shown in Table 2.

For microstate parameter duration of microstate D, result of one-way ANOVA found that there was statistically significant difference between groups, $\mathrm{F}(3,145)=3.141, p=0.027<0.05$. A LSD post hoc test revealed that duration of microstate $\mathrm{D}$ was statistically significantly higher in the right motor disturbance group $(45.41 \pm 9.17, p=0.004)$ and no motor disturbance group (46.88 $\pm 9.53, p=0.019)$ compared to the left motor disturbance group $(51.71 \pm 10.76)$ as shown in Table 2.

\section{Results of Classification}

In this study, we considered classifying a subject into one of two classes, the awake and somnolent subjects (124) as class 1, and subjects with stupor and coma (28) as class 2. We also classified the awake (95) and DoC (57) class, however, the classification performance was not good (the highest accuracy was below 80\%). Therefore, the following results shew the classification of DoC patients (consisting of stupor, light coma, middle coma, and deep coma) and awake controls (consisting of wakefulness and somnolence). 
In our classification, the inputs to each classifier included four microstate parameters 'duration', 'occurrence', 'coverage' and 'GFP' as classification features from each microstate. The state of consciousness (DoC or wakefulness) was also inputted as a label to the classifier.

In the following, we will show the classification results from SVM and AdaBoost as two benchmark classifiers. Then, we will present the classification results from our ensemble classifier EOSVM.

\section{Classification Results from SVM}

First of all, we use a single SVM as a benchmark to classify the positive cases (DoC subjects) and negative cases (no DoC subjects) on three datasets: the original dataset, random over-sampling dataset and random under-sampling dataset. To achieve a stable result, it is executed 30 times for features from the different number of microstates $(2,4,6,8,10,12)$.

Figure 6 shows the accuracy, sensitivity, specificity and F1-score from classifier SVM. The results of the original dataset are depicted in blue box plots. It is seen from these box plots that the overall specificity for the different number of microstates features is around $90 \%$, which is reasonably good. However, the results of sensitivity and F1-Score for the different number of microstates features are under $50 \%$ and $60 \%$, respectively, which are poor for a clinically confident diagnosis decision. Thus, the classification over the original samples with heavy imbalance is not acceptable from the benchmark SVM classifier.
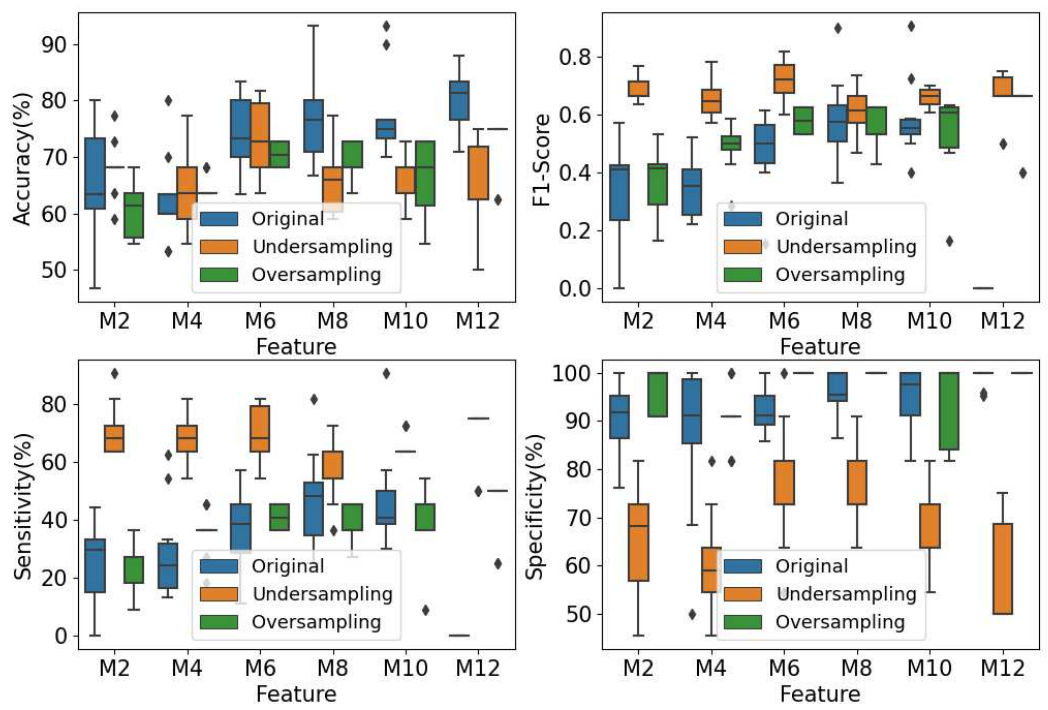

Figure 6. Classification results from SVM. $M+$ number on the horizontal axis refers to how many microstate maps the features are derived from. For example, M2 refers to the features derived from 2 microstate maps.

One may think that the poor performance in sensitivity and F1-score results from data imbalance. We remove data imbalance in the original training set through random under-sampling and random oversampling, respectively. Random under-sampling reduces the number of majority class subjects to match the minority class count. And Random over-sampling increases the number of minority class subjects to match the majority class count. Then, we evaluate the classification performance of the SVM trained with the under-sampled and over-sampled datasets, respectively.

The classification results of 30 runs for features from the different number of microstates are shown in the green and orange box plots of Figure 6. The orange box plots are derived from under-sampling, while the green box plots are obtained from over-sampling. It is seen from these green and orange box plots that

- Random under-sampling does improve the performance of F1-score and sensitivity; and

- Random over-sampling improves the specificity performance.

Although the performance of sensitivity and F1-Score from under-sampled datasets has improved significantly, the accuracy from random under-sampling is statistically poor, mostly below $70 \%$. Therefore, the SVM classifier does not give satisfactory classification results over the balanced data constructed from the original imbalanced data through either random under-sampling or random over-sampling. Further improvement is required, and will be achieved through our EOSVM.

\section{Classification Results from AdaBoost}

As AdaBoost is one of the most efficient machine learning method in recent years ${ }^{15,16}$, we employ classifier AdaBoost as another benchmark to classify the positive cases (DoC subjects) and negative cases (no DoC subjects) in our experiments. Similarly, the 
classifier is executed 30 times to get a stable result and the three datasets (the original dataset, random over-sampling dataset and random under-sampling dataset) are investigated.

Figure 7 shows the accuracy, sensitivity, specificity and F1-score from classifier AdaBoost. The results of the original dataset are depicted in the blue box plots. From these box plots, high specificity, low sensitivity, and low F1-Score are observed, which are similar to those in the benchmark classifier SVM in figure 6. Even worse, the sensitivity from most microstates features tends to zero in some runs, implying that the benchmark classifier AdaBoost classifiies all samples to the majority class, i.e., negative cases.

Also, the random under- and random over-sampled datasets are respectively classified by using AdaBoost. The classification results of 30 runs are shown in the green (random over-sampling) and orange (random under-sampling) box plots of Figure 6 . It is seen from these green and orange box plots that

- Random under-sampled datasets lead to the predictions of all the cases to be in the majority class (i.e., negative cases; and

- Random over-sampling improves the performance of F1-score and sensitivity.

However, the improved sensitivity performance is still statistically poor, mostly below $60 \%$. Also, the accuracy is sacrificed when AdaBoost is trained with either random under-sampling or random over-sampling. From these results, it is seen that the classifier AdaBoost predicts samples to be in the majority class more seriously than the classifier SVM does. Therefore, AdaBoost does not give better performance on our imbalanced dataset than the classifier SVM does.
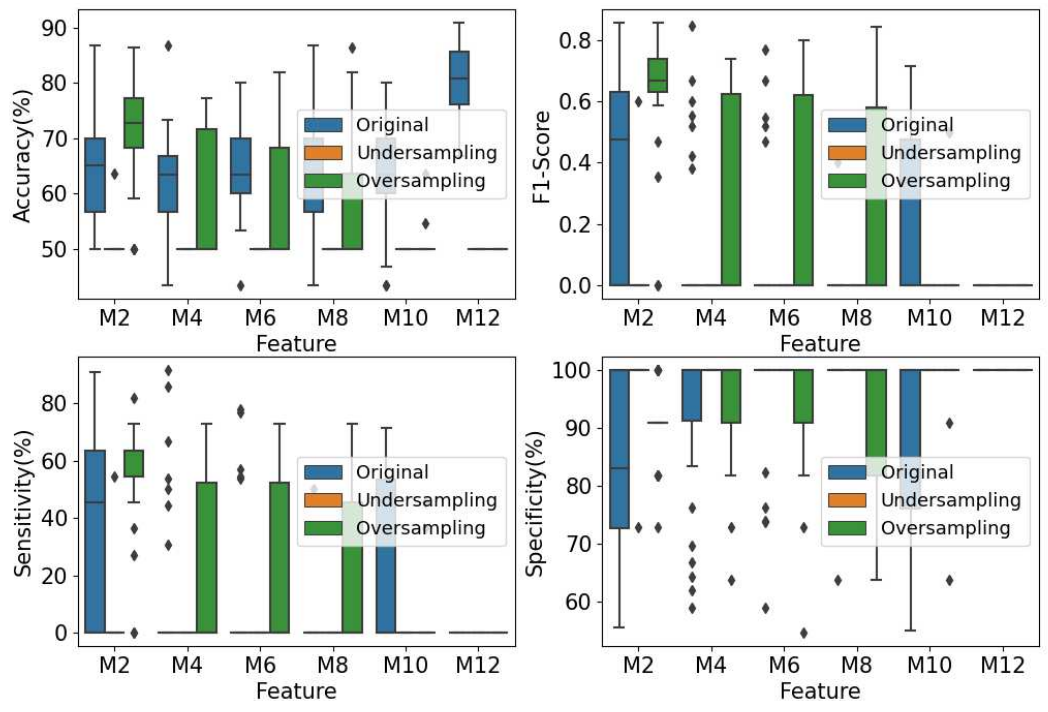

Figure 7. Classification results from AdaBoost. $M+$ number on the horizontal axis refers to how many microstate maps the features are derived from. For example, M4 refers to the features derived from 4 microstate maps.

\section{Classification Results from EOSVM}

For the classification from EOSVM, we present the results from EOSVM consisting of 100 SVMs because the performance under this setting is better than that under other settings of 10,20,50, and $150 \mathrm{SVMs}$ in our experiments. When EOSVM is trained, each SVM in the EOSVM gives a prediction. Altogether, 100 predictions are obtained from the 100 SVMs of EOSVM. These 100 predictions may be the same or different. Thus, they are fused to give the final prediction. In this study, the fusion is carried out through a simple majority voting. Different settings of the majority voting are tested ranging from over $50 \%$ up to $100 \%$ majority voting.

\section{Classification result based on the first group of evaluationmetrics}

The impact of the number of microstate maps on the performance of the EOSVM classification was investigated. The results were used to develop a guideline for the selection of subject features to classify subjects to DoC or wakefulness.

The EOSVM was trained with different numbers of microstate features. In this study, the settings of 2, 4, 6, 8, 10 and 12 microstate maps were considered for EOSVM training. For each of these settings, the EOSVM classifier was trained 30 times. The classification results from the trained EOSVM were depicted in Figure 8. The blue, orange, and green box plots refer to the classification results of EOSVM from 50\% majority voting, 90\% majority voting, and 100\% majority voting, respectively. 

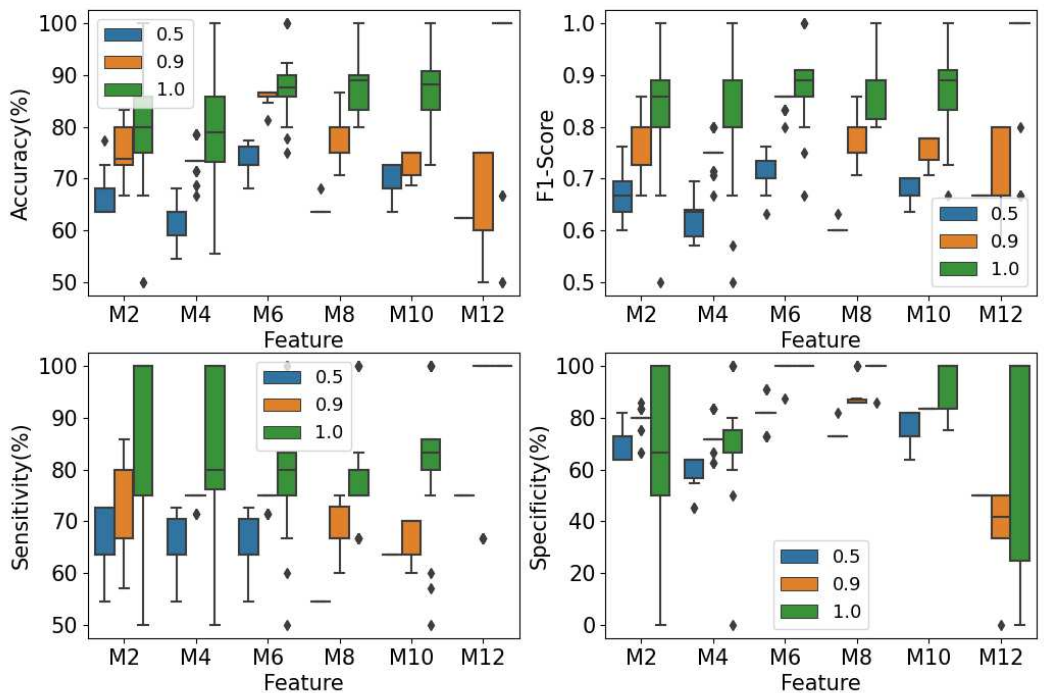

Figure 8. Classification results from EOSVM. $M+$ number on the horizontal axis refers to how many microstate maps the features are derived from. For example, M6 refers to the features derived from 6 microstate maps.

It is observed from the green box plots in Figure 8 that with the requirement of a consensus from all SVMs (i.e., 100\% majority voting), the classification accuracy in the scenario of 6, 8 and 10 microstates all give about $89 \%$ accuracy of classification and prediction. Meanwhile, the F1-Score from 6 microstate maps and 10 microstates maps are around 89\%. These performance metrics have been improved significantly in comparison with the results from a single SVM classifier (Figure 6) and AdaBoost classifier (Figure 7).

However, what is the percentage of the patients that can be diagnosed in or out DoC with the accuracy of about $89 \%$ ? This is another issue that can be captured by our second group of three metrics defined in Equations (7), (9), and (8). This will be investigated later in Section .

The orange box plots in Figure 8 illustrate the classification results when a 90\% majority voting rule is employed, which means that at least $90 \%$ of the SVMs in the EOSVM classifier give the same classification to DoC group, awake controls, or NotSure class. An agreement from a $90 \%$ majority voting is considered to have the credibility to give a medical diagnosis with confidence. It is seen from Figure 8 that approximately 85\% accuracy and F1-Score measures are achieved for 6 microstates. This is the best performance among all the settings of microstate maps, indicating the potential of 6 microstates in the diagnosis of DoC.

The blue box plots in Figure 8 show the classification results when a simple 50\% voting rule is applied. It is seen from the figure that all performance metrics are lower than those under $90 \%$ majority voting and $100 \%$ majority voting. Nevertheless, the performance with the features from 6 microstate maps is still the best. Together with our analysis above on the settings of the number of microstates, we conclude that the setting of 6 microstates is best suitable for the classification of stroke patients.

\section{Classification results based on the second group of evaluation metrics}

We evaluated the second group of three metrics defined in Equations (7), (9) and (8). The evaluation was carried out with a varying number of microstate maps. The number of microstate maps took the values of $2,4,6,8,10$ and 12 . The voting rule varied from over 50\% majority voting to $100 \%$ majority voting of the SVMs in the EOSVM classifier. Some evaluation results were depicted in Figure 9.

With 100\% majority voting from all SVMs in the EOSVM classifier, all classifications to DoC or wakefulness were correct for 2, 4, 6, 8 and 10 microstate maps. This was indicated in the TruePrediction results shown in Figure 9(a). The TruePrediction value reached its maximum of $44.84 \%$ under 10 microstate maps. The values of metrics in the first group in this situation were also reasonable (with accuracy of $88.03 \%, \mathrm{~F} 1$-Score of 0.88 , sensitivity of $82.61 \%$ and specificity of $94.5 \%$ ). The remaining $55.12 \%$ of the patients were classified into the NotSure class, meaning that the classifier was not able to draw a conclusion about whether or not the patients were DoC patients or awake controls. Traditional clinic examinations were needed for the assessment of these patients.

With a 90\% majority voting rule, Figure 9(b) shows that the TruePrediction reaches its maximum of $65.91 \%$ under 6 microstate maps. In this situation, $34.09 \%$ of patients were classified into the NotSure class. Therefore, among the patients who get a clear final prediction, $85.96 \%$ of the prediction is true (the accuracy is $85.96 \%$ ) and the F1-Score is $85.13 \%$.

Figure 9(c) depicts the scenario when an over 50\% majority voting rule is applied. All patients could be classified (NotSure 


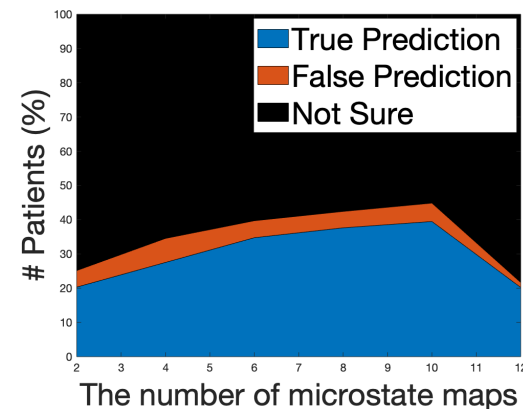

(a)

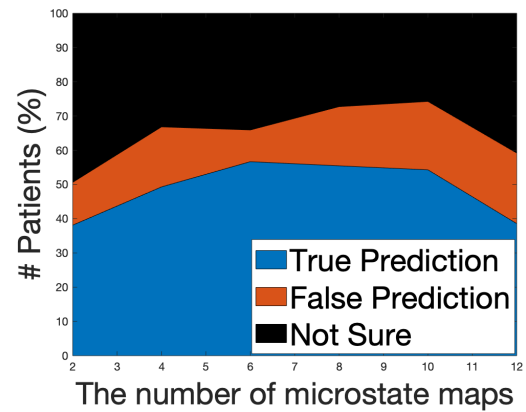

(b)

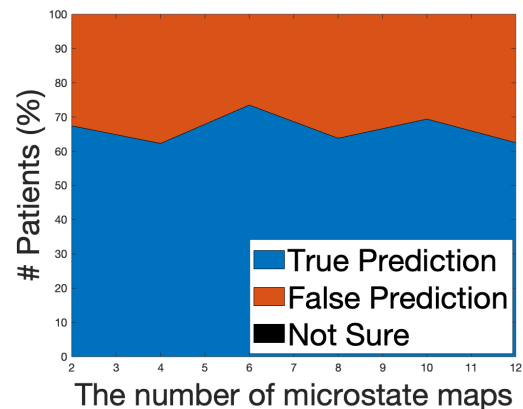

(c)

Figure 9. Evaluation results of TruePrediction, FalsePrediction, and NotSure under different numbers of microstate maps. (a) represents the $100 \%$ majority voting results, (b) represents the $90 \%$ majority voting results, (c) represents the $50 \%$ majority voting results. The black area represents the proportion of patients that cannot be predicted (NotSure), giving no final prediction from the EOSVM. The red area stands for FalsePrediction from the EOSVM, i.e., either classifies a DoC patient to the wakefulness class or predicts an awake patient to the DoC class. The blue area refers to TruePredictionin percentage, i.e., classifies DoC and awake patients correctly to their respective classes.

$=0$ ). The TruePrediction was significantly high (over 60\%). However, the FalsePrediction was also clearly significant (over $30 \%$ ). Thus, useful information was not obtained from these results for medical assessments of stroke patients. Thus, a simple over $50 \%$ majority voting rule was not applicable in the scenarios that we investigated in this study.

\section{Further Discussions}

\section{Statistical Analysis}

EEG microstates reflect the states of consciousness in stroke patients. Through correlation analysis, it has been found that the occurrence, duration, and coverage of microstate $\mathrm{F}$ all increase significantly when the consciousness level of a stroke patient becomes worse. In other words, stroke subjects with DoC have higher values of the three parameters of microstate $\mathrm{F}$ than those who are awake. Similarly, the duration of microstates A, B and C is higher in the subjects with DoC than those who are awake. In contrast, the occurrence of microstates $\mathrm{B}, \mathrm{C}$, and $\mathrm{D}$ are all lower in stroke patients with $\mathrm{DoC}$ than those who are awake. In terms of the coverage of the microstates, microstates $\mathrm{A}$ and $\mathrm{F}$ show an increasingly dominant position in stroke patients with DoC compared to awake subjects. However, microstate $\mathrm{D}$ is dominant in awake subjects.

In comparison with previous studies on consciousness and microstates, there were some similar consequences. When the four canonical EEG microstates in sleep stages and in waking states were compared by Brodbeck and colleagues ${ }^{25}$, their results showed that microstate $\mathrm{C}$ was dominant in awake states and sleep stages $\mathrm{N} 1$ and N3, but microstate B dominated in sleep stage N2. Also, the duration of all four microstates increased in sleep stage N3. Similar to these results, our study showed that the duration of $\mathrm{A}, \mathrm{B}$ and $\mathrm{C}$ increased in subjects who were in coma compared with those who were awake. This could be partly explained by the incidence of slow waves in the EEG data.

Katayama and colleagues explored changes in EEG microstates in participants undergoing hypnosis ${ }^{26}$. Their analysis demonstrated that the duration and occurrence of microstates B and D decreased during hypnosis relative to rest which supported the notion that microstate $\mathrm{D}$ was associated with attention and decreased in cognitive control during hypnosis ${ }^{27}$. Similar to these results, our study revealed that the occurrence of microstates B and D decreased in subjects with DoC relative to wakefulness.

EEG microstates also reflect the motor disturbance in stroke patients. One-way ANOVA demonstrates that there are significant differences in the occurrence of microstate $\mathrm{A}$ and duration of microstate $\mathrm{D}$ between the different motor disturbance groups. It is worth noting that the occurrence of microstate A is remarkably higher in the right motor disturbance group than that in the left motor disturbance group, however, the duration of microstate D is significantly higher in the left motor disturbance group than that in the right motor disturbance group. These results show a similar agreement with the report in the pioneering study in ${ }^{14}$ on the duration of microstate $\mathrm{D}$ in stroke. This previous study showed that in stroke patients, the microstate $\mathrm{C}$ and $\mathrm{D}$ differed in their duration after both a left and a right lesion concerning controls $(\mathrm{C}$ was lower than $\mathrm{D}$ in the left, and $\mathrm{D}$ was lower than $\mathrm{C}$ in the right lesion) $)^{14}$. Therefore, the stroke lesions in the brain and motor disturbance after stroke seemed to be both related to the duration of microstate $\mathrm{D}$.

\section{Classification of DoC Patients and Awake Controls}


The dataset in this study is an imbalanced dataset with 57 positive cases (DoC subjects) and 95 negative cases (no DoC subjects). For this kind of heavily imbalanced dataset, common classifiers always predict the minor class as the major class. As a result, the sensitivity of the classification is very low. However, in clinical diagnosis, a test with high sensitivity is necessary for ruling out disease ${ }^{28}$. Sensitivity in this study refers to the ability of the analysis to correctly detect patients with DoC who do have DoC (i.e., true positives).

From Figures 6 and 7, it is seen that the classification results from classifiers SVM and AdaBoost have poor sensitivity performance of lower than $60 \%$. However, the sensitivity from our classifier EOSVM has been improved significantly to above $80 \%$. Therefore, the design of the EOSVM successfully improved the sensitivity of classification in the imbalanced dataset which is useful in clinical application. Also, the classifier EOSVM in this study improved the detection of the patients with DoC comparing with the classifier SVM and Adaboost.

As this paper focuses on the study of the classification of stroke patients in a hospital, a test with high sensitivity is useful for ruling out disease ${ }^{28}$. Sensitivity in this study refers to the ability of the analysis to correctly detect patients with DoC who do have DoC (i.e., true positives). From Figures 6 and 7, it is seen that the classification results from classifiers SVM and AdaBoost have poor sensitivity performance of lower than $60 \%$. However, the sensitivity from our classifier EOSVM has been improved significantly to above $80 \%$. Therefore, the design of the EOSVM successfully improved the detection of the patients with DoC rather than the awake controls.

The performance of classification between DoC patients and awake controls in the EOSVM demonstrated that microstate parameters were effective features to classify awake controls and DoC patients. We compared the classification results under different numbers of microstate maps and different majority voting rules in the EOSVM. The results showed that when EEG data were clustered into 6 clusters, the EEG parameters contributed to better classification performance. These results also supported the hypothesis that there might be more than four primary microstates 6,7 .

\section{Conclusion}

Our study explores the relationship between EEG microstates and the clinical states including the consciousness and motor disturbance in stroke patients. Statistical analysis reveals that the occurrence of microstate A and duration of microstate D are correlated with the sides of the motor disturbance in stroke patients. Microstates A, B, C, D, and F are all correlated with the states of consciousness in different parameters. Further classification of stoke patients to DoC or wakefulness from an EOSVM classifier demonstrates that about $65.91 \%$ of stroke patients could be predicted with an accuracy of over $85 \%$. Therefore, EOSVM classification based on EEG microstates is helpful to medical practitioners in DoC detection with saved resources that would otherwise be consumed in traditional clinic checks.

\section{References}

1. Sousa, R. M. et al. Contribution of chronic diseases to disability in elderly people in countries with low and middle incomes: a 10/66 dementia research group population-based survey. The Lancet 374, 1821-1830 (2009).

2. Reinkensmeyer, D. J. et al. Understanding and treating arm movement impairment after chronic brain injury: progress with the arm guide. (2014).

3. Claassen, J. et al. Bedside quantitative electroencephalography improves assessment of consciousness in comatose subarachnoid hemorrhage patients. Annals neurology 80, 541-553 (2016).

4. Satoko et al. Gait-synchronized rhythmic brain stimulation improves poststroke gait disturbance: A pilot study. Stroke 50, 3205-3212 (2019).

5. González, B., Rodríguez, M., Ramirez, C. \& Sabaté, M. Disturbance of motor imagery after cerebellar stroke. Behav. Neurosci. 119, 622-626 (2005).

6. Seitzman, B. A. et al. Cognitive manipulation of brain electric microstates. Neuroimage 146, 533-543 (2017).

7. Michel, C. M. \& Koenig, T. EEG microstates as a tool for studying the temporal dynamics of whole-brain neuronal networks: a review. Neuroimage 180, 577-593 (2018).

8. Santarnecchi, E. et al. EEG microstate correlates of fluid intelligence and response to cognitive training. Brain Topogr. 30, 502-520 (2017).

9. Irisawa, S. et al. Increased omega complexity and decreased microstate duration in nonmedicated schizophrenic patients. Neuropsychobiology 54, 134-139 (2006).

10. Nishida, K. et al. EEG microstates associated with salience and frontoparietal networks in frontotemporal dementia, schizophrenia and alzheimer's disease. Clin. Neurophysiol. 124, 1106-1114 (2013). 
11. Andreou, C. et al. Resting-state connectivity in the prodromal phase of schizophrenia: insights from eeg microstates. Schizophr. Res. 152, 513-520 (2014).

12. Corradini, P. L. \& Persinger, M. A. Spectral power, source localization and microstates to quantify chronic deficits from 'mild'closed head injury: Correlation with classic neuropsychological tests. Brain Inj. 28, 1317-1327 (2014).

13. Drissi, N. M. et al. Altered brain microstate dynamics in adolescents with narcolepsy. Front. Hum. Neurosci. 10, 369 (2016).

14. Zappasodi, F. et al. Prognostic value of EEG microstates in acute stroke. Brain Topogr. 30, 698-710 (2017).

15. Bergstra, J., Casagrande, N., Erhan, D., Eck, D. \& Kegl, B. Aggregate features and adaboost for music classification. Mach. Learn. 65, 473-484 (2006).

16. Yang, Y. et al. Performance comparison of gestures recognition system based on different classifiers. IEEE Transactions on Cogn. Dev. Syst. (2020).

17. Wang, F. et al. Using phase synchrony index for improved assessment of consciousness in ischemic stroke patients. IEEE Access (2019).

18. Pascual-Marqui, R. D., Michel, C. M. \& Lehmann, D. Segmentation of brain electrical activity into microstates: model estimation and validation. IEEE Transactions on Biomed. Eng. 42, 658-665 (1995).

19. Pascual-Marqui, R. D. \& Michel, C. M. Segmentation of brain electrical activity into microstates: model estimation and validation. IEEE transactions on bio-medical engineering 42, 658-65 (1995).

20. Poulsen, A. T., Pedroni, A., Langer, N. \& Hansen, L. K. Microstate EEGlab toolbox: An introductory guide. bioRxiv 289850 (2018).

21. Hernandez, L. D., Rieger, K., Baenninger, A., Brandeis, D. \& Koenig, T. Towards using microstate-neurofeedback for the treatment of psychotic symptoms in schizophrenia. a feasibility study in healthy participants. Brain Topogr. 29, 308-321 (2016).

22. Gschwind, M. et al. Fluctuations of spontaneous EEG topographies predict disease state in relapsing-remitting multiple sclerosis. NeuroImage: Clin. 12, 466-477 (2016).

23. Grieder, M. et al. Discovering EEG resting state alterations of semantic dementia. Clin. neurophysiology 127, 2175-2181 (2016).

24. Custo, A. et al. Electroencephalographic resting-state networks: source localization of microstates. Brain Connect. 7, 671-682 (2017).

25. Brodbeck, V. et al. EEG microstates of wakefulness and NREM sleep. Neuroimage 62, 2129-2139 (2012).

26. Katayama, H. et al. Classes of multichannel EEG microstates in light and deep hypnotic conditions. Brain Topogr. 20, $7-14$ (2007).

27. Xu, X., Yuan, H. \& Lei, X. Activation and connectivity within the default mode network contribute independently to future-oriented thought. Sci. Reports 6, 21001 (2016).

28. Altman, D. G. \& Bland, J. M. Diagnostic tests. 1: Sensitivity and specificity. BMJ: Br. Med. J. 308, 1552 (1994).

\section{Acknowledgements}

This work was supported in part by the Scientific Research Foundation of XiHua University under Grant RZ2100000758, the Australian Research Council (ARC) through the Linkage Project Scheme under Grant LP140100394 and the Wu Jieping Medical Foundation of China under Grant 320675016129.

\section{Data availability}

The datasets used during the current study are available from the corresponding author on reasonable request.

\section{Competing interests}

The authors declare no competing interests. 